\title{
An Algebraic Approach to Discrete Mechanics
}

\author{
John C. Baez and James W. Gilliam \\ Department of Mathematics \\ University of California \\ Riverside, California 92521
}

December 13, 1993

Published in Lett. Math. Phys. 31 (1994), 205-212.

\begin{abstract}
Using basic ideas from algebraic geometry, we extend the methods of Lagrangian and symplectic mechanics to treat a large class of discrete mechanical systems, that is, systems such as cellular automata in which time proceeds in integer steps and the configuration space is discrete. In particular, we derive an analog of the Euler-Lagrange equation from a variational principle, and prove an analog of Noether's theorem. We also construct a symplectic structure on the analog of the phase space, and prove that it is preserved by time evolution.
\end{abstract}

\section{Introduction}

One of the first uses of digital computers was to approximately simulate physical systems by numerically solving differential equations. As physicists become more familiar with digital computation, there has naturally been increased interest in exact simulation of "discrete mechanical systems," in which time evolution proceeds in integer steps and the state space is a finite set. (We wish to distinguish such systems from "discrete dynamical systems" in which time is discrete but the state space is a manifold.) Presently, the most widely studied examples of discrete mechanical systems are cellular automata on finite lattices. But there is no need to limit discrete 
mechanics to the study of cellular automata, which are analogous to field theories in classical mechanics. Just as it is useful to treat classical field theories as a special case of classical mechanical systems, it may be useful to treat cellular automata as a special case of discrete mechanical systems.

It seems worthwhile to attempt to extend standard techniques in classical mechanics, such as the Lagrangian and Hamiltonian formalisms, to discrete mechanics. The lack of a notion of differentiation might appear an insurmountable obstacle to such a project. We show, however, that the algebraic definition of differential forms provides a partial substitute for the usual differential calculus. We begin by deriving an analog of the Euler-Lagrange equation for discrete mechanics, starting from a variational principle. As an example of how this analog works, we prove a version of Noether's theorem applicable to this context. We also relate our framework to Hamiltonian mechanics, or more precisely, symplectic geometry.

For somewhat related work on extending notions of classical mechanics to more general algebraic settings see, for example [7, 10]. For related work in discrete dynamical systems see $[2,12]$ and the references therein. For applications of symplectic mechanics to cellular automata see [3]. The authors would like to thank Norm Margolus, Bruce Smith, Mark Smith and Tomaso Toffoli for many useful discussions of cellular automata.

\section{The Euler-Lagrange Equation}

In order to apply the methods of differential geometry to discrete configuration spaces, we will use the strategies of algebraic geometry. This is in fact quite natural, given the successful application of algebraic geometry to many problems involving integrable systems $[6,8]$.

The key idea is to replace the real numbers by an arbitrary commutative ring $k$, with the most interesting examples for the purposes of discrete mechanics being the integers, the integers modulo $n$, and other finite rings. Also, rather than working 
directly with the configuration space, we work with the algebraic functions on the configuration space, which form a commutative algebra $A$ over $k$. For example, if $k$ is a field and the configuration space is an $n$-dimensional vector space over $k$, we have $A=k\left[x_{1}, \ldots, x_{n}\right]$, the algebra of polynomial functions in $n$ variables. Since the theory may seem rather abstract, in what follows we illustrate each construction with the simplest example, the particle in a potential in a one-dimensional configuration space, where $A=k[x]$.

Let $k$ be a commutative ring and $A$ a commutative algebra over $k$. Just as $A$ plays the role of the functions on configuration space, the algebra $H=A^{\otimes(T+1)}=A \otimes \cdots \otimes A$ is to be thought of as the functions on the space of "histories," where time takes values in the discrete set $\{0, \ldots, T\}$. It will be convenient to sometimes use the notation $H=A_{0} \otimes A_{1} \otimes \cdots \otimes A_{T}$, where the algebras $A_{i}$ are simply copies of $A$, with $A_{i}$ thought of as the functions on configuration space at time $i$.

Fix an element $\mathcal{L} \in A \otimes A$, which will play the role of the Lagrangian for our system. In the algebra $H$ let

$$
S=\sum_{i=0}^{T-1} \mathcal{L}_{i}
$$

where $\mathcal{L}_{i}=1 \otimes \cdots \otimes \mathcal{L} \otimes \cdots \otimes 1$ with $\mathcal{L}$ occupying the $i$ th and $(i+1)$ st slots. The element $S$ corresponds to the action functional in classical mechanics.

Example: Suppose 2 is a unit in $k$ and the algebra $A=k[x]$, so $A \otimes A \cong k\left[q_{1}, q_{2}\right]$ and $H \cong k\left[q_{0}, \ldots, q_{T}\right]$, the polynomials in $T+1$ variables over $k$. We then consider the Lagrangian $\mathcal{L}_{i}$ for a particle in a polynomial potential $V$ as a function of consecutive positions $q_{i}$ and $q_{i+1}$ of the particle,

$$
\mathcal{L}_{i}=\mathcal{L}\left(q_{i}, q_{i+1}\right)=\frac{1}{2} m \dot{q}_{i}^{2}-V\left(q_{i}\right)
$$

where we define $\dot{q}_{i}=q_{i+1}-q_{i}$ and where $m$ is a ring element representing the mass of the particle.

In order to formulate a variational principle we need an algebraic analog of the the differential calculus. For this we borrow from algebraic geometry the concept of 
Kähler differentials $[1,5]$, or more generally, algebraic differential forms. If $B$ is a commutative $k$-algebra, the algebraic differential forms $\Omega(B)$ are a graded-commutative differential graded algebra with $\Omega^{0}(B)=B$, defined by the following universal property: given any graded-commutative differential graded algebra $\Omega$ and given any algebra homomorphism $f: B \rightarrow \Omega^{0}$, there exists a unique differential graded algebra homomorphism $f_{*}: \Omega(B) \rightarrow \Omega$ such that the following diagram commutes:

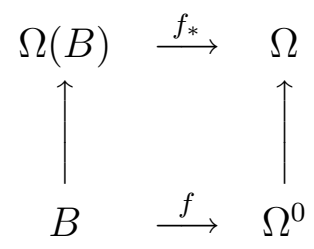

We write the product in $\Omega(B)$ as a wedge product and the differential as $d$. Concretely, $\Omega(B)$ is the algebra generated by $B$ and elements $d a$, where $a \in B$, with the relations:

$$
\begin{gathered}
d(\lambda a)=\lambda d a, \quad d(a+b)=d a+d b, \quad d(a b)=d a \wedge b+a \wedge d b, \\
a \wedge d b=d b \wedge a, \quad d a \wedge d b=-d b \wedge d a, \quad d a \wedge d a=0 .
\end{gathered}
$$

(The last is only necessary if 2 is not a unit in $k$.)

Now it can be shown that for any algebra $A$,

$$
\Omega^{p}(A \otimes A)=\bigoplus_{q=0}^{p} \Omega^{q}(A) \otimes \Omega^{p-q}(A) .
$$

so by induction,

$$
\Omega^{1}(H)=\bigoplus_{i=0}^{T} A_{0} \otimes \cdots \otimes \Omega^{1}\left(A_{i}\right) \otimes \cdots \otimes A_{T}
$$

Let $p_{i}: \Omega^{1}(H) \rightarrow \Omega^{1}(H)$ denote projection onto the $i$ th summand. We define $d_{i}=p_{i} d$ and note $d=\sum_{i=0}^{T} d_{i}$. This allows us to define a new operator $\delta=\sum_{i=1}^{T-1} d_{i}$, which we think of as a variation keeping the endpoints fixed. We now determine the variation of the action $S$ with fixed endpoints.

Proposition 1. $\delta S=\sum_{i=1}^{T-1} d_{i} \mathcal{L}_{i}+d_{i} \mathcal{L}_{i-1}$. 
Proof - For $j \neq i, i+1, d_{j} \mathcal{L}_{i}=0$, hence

$$
\delta S=\delta \sum_{i=0}^{T-1} \mathcal{L}_{i}=\sum_{i=0}^{T-1} \delta \mathcal{L}_{i}=\sum_{i=1}^{T-1} d_{i} \mathcal{L}_{i}+d_{i} \mathcal{L}_{i-1}
$$

Example: Consider the Lagrangian of the previous example. Then

$$
\begin{aligned}
\delta S & =\delta \sum_{i=0}^{T-1} \mathcal{L}\left(q_{i}, q_{i+1}\right) \\
& =\sum_{i=0}^{T-1} \delta\left(\frac{1}{2} m \dot{q}_{i}^{2}\right)-\delta V\left(q_{i}\right) \\
& =\sum_{i=1}^{T-1}\left(\left(-m\left(\dot{q}_{i}-\dot{q}_{i-1}\right)-V^{\prime}\left(q_{i}\right)\right) \delta q_{i}\right)-\left(m \dot{q}_{0}+V^{\prime}\left(q_{0}\right)\right) \delta q_{0}+m \dot{q}_{T-1} \delta q_{T} \\
& =\sum_{i=1}^{T-1}\left[-m\left(\dot{q}_{i}-\dot{q}_{i-1}\right)-V^{\prime}\left(q_{i}\right)\right] \delta q_{i}
\end{aligned}
$$

where in the last step we use $\delta q_{0}=\delta q_{T}=0$. In what follows we make precise the sense in which the vanishing of $\delta S$ yields the equation of motion

$$
m\left(\dot{q}_{i}-\dot{q}_{i-1}\right)=-V^{\prime}\left(q_{i}\right)
$$

This is the discrete analog of Newton's equation of motion for a particle moving in a potential, $m \ddot{q}=-V^{\prime}(q)$.

The vanishing of the expression $d_{i} \mathcal{L}_{i}+d_{i} \mathcal{L}_{i-1}$ is supposed to correspond to the Euler-Lagrange equation in this framework. But we must describe precisely in what sense it vanishes. It certainly does not vanish as a 1-form on the whole space of histories; rather, it should only vanish on trajectories satisfying the equations of motion. In the discrete-time context, the equations of motion express the configuration at a particular time as a function of the configurations at the two preceding times. We formalize this in terms of a homomorphism $\varphi: A_{2} \rightarrow A_{0} \otimes A_{1}$. Such a homomorphism determines a homomorphism $\Phi: A_{1} \otimes A_{2} \rightarrow A_{0} \otimes A_{1}$ by

$$
\begin{aligned}
a \otimes 1 & \mapsto 1 \otimes a \\
1 \otimes a & \mapsto \varphi(a) .
\end{aligned}
$$


which plays the role of a time evolution map. (If it is puzzling that the indices decrease by 1 in this map, simply recall that a map from a space $X$ to a space $Y$ allows one to pull back functions on $Y$ to functions on $X$.) We say that $\varphi$, or alternatively $\Phi$, satisfies the Euler-Lagrange equation provided

$$
\Phi_{*} d_{1} \mathcal{L}_{1}+d_{1} \mathcal{L}_{0}=0
$$

where $\Phi_{*}: \Omega^{1}\left(A_{1} \otimes A_{2}\right) \rightarrow \Omega^{1}\left(A_{0} \otimes A_{1}\right)$ is the map induced by $\Phi$, and we regard $A_{0} \otimes A_{1}$ and $A_{1} \otimes A_{2}$ as subalgebras of $H$ in order to define the differential $d_{1}$.

Example: Continuing the example above, and assuming that $m$ is a unit in $k$, define $\varphi$ by

$$
\varphi\left(q_{2}\right)=q_{1}+\dot{q}_{0}-m^{-1} V^{\prime}\left(q_{1}\right)
$$

This map expresses the particle's position at time 2 as a function of its positions at times 0 and 1 . Let us show $\varphi$ satisfies the equations of motion. We have $A_{0} \otimes A_{1} \cong$ $k\left[q_{0}, q_{1}\right], A_{1} \otimes A_{2} \cong k\left[q_{1}, q_{2}\right]$, and $\Phi: k\left[q_{1}, q_{2}\right] \rightarrow k\left[q_{0}, q_{1}\right]$ satisfies

$$
\begin{gathered}
\Phi\left(q_{1}\right)=q_{1}, \quad \Phi\left(q_{2}\right)=\varphi\left(q_{2}\right)=2 q_{1}-q_{0}-m^{-1} V^{\prime}\left(q_{1}\right), \\
\Phi_{*}\left(d q_{1}\right)=d q_{1}, \quad \Phi_{*}\left(d q_{2}\right)=2 d q_{1}-d q_{0}-m^{-1} V^{\prime \prime}\left(q_{1}\right) d q_{1}
\end{gathered}
$$

SO

$$
\begin{aligned}
\Phi_{*} d_{1} \mathcal{L}_{1}+d_{1} \mathcal{L}_{0}= & \Phi_{*} \partial_{q_{1}} \mathcal{L}\left(q_{1}, q_{2}\right) d q_{1}+\partial_{q_{1}} \mathcal{L}\left(q_{0}, q_{1}\right) d q_{1} \\
= & \Phi_{*}\left(\left(-m\left(q_{2}-q_{1}\right)-V^{\prime}\left(q_{1}\right)\right) d q_{1}\right)+m\left(q_{1}-q_{0}\right) d q_{1} \\
= & \left(-m\left(2 q_{1}-q_{0}-m^{-1} V^{\prime}\left(q_{1}\right)-q_{1}\right)-V^{\prime}\left(q_{1}\right)\right) d q_{1} \\
& +m\left(q_{1}-q_{0}\right) d q_{1} \\
= & 0
\end{aligned}
$$

as desired. 


\section{$3 \quad$ Symplectic Geometry}

In continuous-time classical mechanics the phase space is defined to be the cotangent bundle over the configuration space. Here, interestingly, it is the Cartesian product of the configuration space with itself that plays the role of a phase space. Thus, the algebra $A \otimes A$ is the analog of the functions on phase space. To clarify this analogy, we would like to define a symplectic structure on $A \otimes A$ that is preserved by time evolution.

In this context it is more convenient to think of the time evolution map $\Phi$ as a map from a fixed copy of $A \otimes A$ to itself. This requires a certain change in indexing; we say that $\Phi: A_{1} \otimes A_{2} \rightarrow A_{1} \otimes A_{2}$ satisfies the Euler-Lagrange equation if

$$
\Phi_{*} d_{1} \mathcal{L}+d_{2} \mathcal{L}=0
$$

where $\mathcal{L}=\mathcal{L}_{1} \in A_{1} \otimes A_{2}$. Note that $d \mathcal{L}=d_{1} \mathcal{L}+d_{2} \mathcal{L}$. We define the closed 2-form $\omega \in \Omega^{2}\left(A_{1} \otimes A_{2}\right)$ by $\omega=-d d_{1} \mathcal{L}=d d_{2} \mathcal{L}$. This plays the role of a symplectic structure on phase space. It is always preserved by time evolution:

Proposition 2. $\omega$ is preserved by the time evolution map $\Phi$, that is, $\Phi_{*} \omega=\omega$, provided $\Phi$ satisfies the Euler-Lagrange equations.

Proof -

$$
\Phi_{*} \omega=-\Phi_{*} d d_{1} \mathcal{L}=-d \Phi_{*} d_{1} \mathcal{L}=d d_{2} \mathcal{L}=\omega
$$

Example: Consider $\mathcal{L} \in A_{1} \otimes A_{2} \cong k\left[q_{1}, q_{2}\right]$. Then

$$
\begin{aligned}
& d_{1} \mathcal{L}=\partial_{q_{1}} \mathcal{L}\left(q_{1}, q_{2}\right) d q_{1} \\
& d_{2} \mathcal{L}=\partial_{q_{2}} \mathcal{L}\left(q_{1}, q_{2}\right) d q_{2}
\end{aligned}
$$

SO

$$
\omega=\partial_{q_{1}} \partial_{q_{2}} \mathcal{L}\left(q_{1}, q_{2}\right) d q_{1} \wedge d q_{2}
$$


Continuing the example of a particle moving in a potential and assuming 2 and $m$ are units, we see that:

$$
\omega=\partial_{q_{1}} \partial_{q_{2}}\left(\frac{1}{2} m\left(q_{2}-q_{1}\right)^{2}-V\left(q_{1}\right)\right) d q_{1} \wedge d q_{2}=m d q_{2} \wedge d q_{1}
$$

If we define the "momentum" $p_{1}$ to be $m \dot{q}_{1}$, we have the familiar formula

$$
\omega=d p_{1} \wedge d q_{1}
$$

Using the time evolution derived above we have, using the new indexing system,

$$
\Phi\left(q_{1}\right)=q_{2}, \quad \Phi\left(q_{2}\right)=2 q_{2}-q_{1}-m^{-1} V^{\prime}\left(q_{2}\right),
$$

so the symplectic structure $\omega$ is preserved:

$$
\begin{aligned}
\Phi_{*} \omega & =\Phi_{*}\left(m d q_{2} \wedge d q_{1}\right) \\
& =m\left(2 d q_{2}-d q_{1}-m^{-1} V^{\prime \prime}\left(q_{2}\right) d q_{2}\right) \wedge d q_{2} \\
& =\omega .
\end{aligned}
$$

Under certain conditions the symplectic structure $\omega$ will be nondegenerate, in the sense that it gives rise to a map from 1-forms on the phase space, that is, $\Omega^{1}(A \otimes A)$, to "vector fields" on phase space, that is, derivations of the algebra $A \otimes A$. In this case there is a full-fledged analog of Hamiltonian mechanics for the theory, $A \otimes A$ becomes a Poisson algebra, and so on. We will not, however, pursue this here.

\section{Noether's Theorem}

Now we consider a simple version of Noether's theorem in this setting. The analog of a vector field on configuration space is a derivation of $A$, that is, a $k$-linear map $v: A \rightarrow A$ map such that $v(a b)=a v(b)+v(a) b$ for all $a, b \in A$. We can construct a derivation $D$ on $A_{1} \otimes A_{2}$ by $D=1 \otimes v+v \otimes 1$; that is,

$$
D(a \otimes b)=a \otimes v b+v a \otimes b
$$


A derivation $v$ is called an infinitesimal symmetry of the Lagrangian $\mathcal{L} \in A_{1} \otimes A_{2}$ provided $(1 \otimes v+v \otimes 1) \mathcal{L}=0$.

Proposition 3. Let $\Phi$ be a time evolution map satisfying the Euler-Lagrange equation and $v$ an infinitesimal symmetry of $\mathcal{L}$, then the quantity $F \in A \otimes A$ defined by $F=-(v \otimes 1) \mathcal{L}$ is preserved by $\Phi$.

Proof - We need to use the pairing between 1-forms and derivations. For any commutative $k$-algebra $B$, there is a unique $B$-bilinear pairing $\langle\cdot, \cdot\rangle: \Omega^{1}(B) \otimes \operatorname{Der}(B) \rightarrow$ $B$ such that $\langle d a, v\rangle=v(a)$ for all $a \in B, v \in \operatorname{Der}(B)$. Also, any isomorphism $f: B \rightarrow$ $C$ of such algebras induces a map $\Phi_{*}: \operatorname{Der}(C) \rightarrow \operatorname{Der}(B)$ given by $\Phi_{*} v=\Phi \circ v \circ \Phi^{-1}$. We thus have

$$
\begin{aligned}
\Phi F & =-\Phi((v \otimes 1) \mathcal{L})=-\Phi\langle d \mathcal{L}, v \otimes 1\rangle=-\Phi\left\langle d_{1} \mathcal{L}, v \otimes 1\right\rangle \\
& =-\left\langle\Phi_{*} d_{1} \mathcal{L}, \Phi_{*}(v \otimes 1)\right\rangle=\left\langle d_{2} \mathcal{L}, \Phi_{*}(v \otimes 1)\right\rangle \\
& =\left\langle d_{2} \mathcal{L}, 1 \otimes v\right\rangle=\langle d \mathcal{L}, 1 \otimes v\rangle=(1 \otimes v) \mathcal{L}=-(v \otimes 1) \mathcal{L}=F
\end{aligned}
$$

Example: Consider the previous example when $V=0$, that is, the free particle. The Lagrangian $\mathcal{L}=\frac{1}{2} m \dot{q}_{1}^{2}=\frac{1}{2} m\left(q_{2}-q_{1}\right)^{2}$ is translation-invariant, that is, it has the derivation $v=\partial_{x}$ as an infinitesimal symmetry:

$$
(v \otimes 1+1 \otimes v)=\partial_{q_{1}}+\partial_{q_{2}}
$$

and

$$
\left(\partial_{q_{1}}+\partial_{q_{2}}\right) \mathcal{L}=0 .
$$

The corresponding conserved quantity is momentum:

$$
F=-(v \otimes 1) \mathcal{L}=-\partial_{q_{1}} \frac{1}{2} m\left(q_{2}-q_{1}\right)^{2}=m \dot{q}_{1} .
$$


Example: Consider a 3 -dimensional configuration space, so that $A=k[x, y, z]$, and let $\mathcal{L}$ be the analog of the Lagrangian for a particle in a spherically symmetric potential. If $k=\mathbb{Z}$ this Lagrangian describes the motion of a particle in the lattice $\mathbb{Z}^{3}$ in discrete time steps. The Lagrangian $\mathcal{L}$ has as infinitesimal symmetries the derivations corresponding to infinitesimal rotations:

$$
x \partial_{y}-y \partial_{x}, \quad y \partial_{z}-z \partial_{y}, \quad z \partial_{x}-x \partial_{z},
$$

so angular momentum is conserved, even though the lattice does not have $S O(3)$ symmetry.

In future work we hope to treat more interesting examples of discrete mechanical systems using Lagrangian and symplectic methods. In particular, as Wolfram [11] has noted, it would be interesting to seek "completely integrable" 1-dimensional cellular automata analogous to the Toda model, KdV equation, and other 1-dimensional completely integrable systems $[6,8]$.

\section{References}

[1] R. Hartshorne, Algebraic Geometry, Springer-Verlag, New York, 1977.

[2] T. Itoh and K. Abe, Discrete Lagrange's equations and canonical equations based on the principle of least action, Appl. Math. Comput. 29 (1989), 161-183.

[3] K. Kaneko, Symplectic cellular automata, Phys. Lett. A 129 (1988) 9-16.

[4] N. Margolus, Physics and computation, MIT Technical Report MIT/LCS/TR415.

Physics-like models of computation, Physica 10D (1984), 81 - 95.

[5] H. Matsumura, Commutative algebra, Benjamin, New York, 1970. 
[6] D. Mumford, An algebro-geometric construction of commuting operators and of solutions to the Toda lattice equation, Korteweg de Vries equation and related non-linear equations, Intl. Symp. on Algebraic Geometry, Kyoto, 1977, pp. 115153.

[7] Y. Nambu, Field theory of Galois' fields, in Quantum Field Theory and Quantum Statistics, edited by I. A. Batalin, C. J. Isham, and G. A. Vilkovisky, Adam Hilger, Bristol, 1987.

[8] Integrable Systems, edited by S. Novikov, London Mathematical Lecture Note Series 60, Cambridge U. P., Cambridge, 1981.

[9] T. Toffoli and N. Margolus, Cellular Automata Machines: a New Environment for Modeling, MIT Press, Cambridge, Mass., 1987.

[10] E. Thiram, D. Verstegen and J. Weyers, p-Adic dynamics, Jour. Stat. Phys. 54 893-913 (1989).

[11] S. Wolfram, personal communication.

[12] A. P. Veselov, What is an integrable mapping?, in What Is Integrability?, V. E. Zakharov, ed., Springer-Verlag, New York, 1991, pp. 251-272. 